\title{
Effect of Xylooligosaccharide Intake on Severe Constipation in Pregnant Women
}

\author{
Ichiro TATEYAMA ${ }^{1}$, Koji HASHII ${ }^{1}$, Ikuo JOHNO ${ }^{1}$, Taeko IINO ${ }^{2}$, Kouichi HIRAI ${ }^{2}$, \\ Yoshihide SuWA ${ }^{3}$ and Yoshinobu KISO ${ }^{2, *}$ \\ ${ }^{1}$ OB/GY Department, Kishiwada City Hospital, Osaka 596-8501, Japan \\ ${ }^{2}$ Institute for Health Care Science, Suntory Ltd., Mishima, Osaka 618-8503, Japan \\ ${ }^{3}$ Quality Assurance Division, Suntory Ltd., Mishima, Osaka 618-8503, Japan
}

(Received September 2, 2004)

\begin{abstract}
Summary Xylooligosaccharides (XOS) are mainly composed of two or three xylose units with $\beta-1,4$ linkages. They are obtained by hemicellulose hydrolysis, which is relatively abundant in the cell walls of grains. XOS increases the number of intestinal Bifidobacterium in humans, and maintains the fecal water content within the normal range. To examine the effect of XOS intake on severe constipation in pregnancy, which is predominant in the third trimester, thirty constipated pregnant women were treated with $4.2 \mathrm{~g}$ XOS daily for 4 wk. During the study, the clinical efficacy was assessed using a daily diary. The subjects indicated the number of stools and the clinical symptom scores. Twenty-nine subjects completed the study. The mean number of stools was $1.1 \pm 0.4$ in the pre-treatment week, and increased in weeks $1-4$ of XOS administration to $5.3 \pm 2.1,5.9 \pm 2.5,6.2 \pm 2.2$ and $6.7 \pm 1.9$, respectively. At the end of the study, 27 subjects could defecate spontaneously. The occurrence of very loose or very hard stools decreased and the stool consistency normalized. The stool color changed from dark to yellowish brown. No side effects were observed. XOS intake was highly effective for the reduction of severe constipation in pregnant women without adverse effects.
\end{abstract}

Key Words xylooligosaccharides, constipation, defecation, pregnant women

Pregnancy can be complicated by bowel irritation, constipation or diarrhea. Anderson reported constipation in $38 \%$ of English women during pregnancy (1). Typical symptoms of constipation are feelings of incomplete defecation, abdominal discomfort, bloating and distention. It is important to improve these symptoms of constipation for the comfort of pregnant women. Severe constipation is predominant in the third trimester of pregnancy. This is due to enlargement of the uterus and pressure on the abdominal and back muscles, prolonging the gastrointestinal transit time (2). However, if drugs are prescribed to resolve the constipation, it is necessary to assure that they do not affect the health of the expectant mother and fetus. Therefore, safe foods or food materials that prevent constipation are desirable for good pregnancy progression. West et al. proposed that the effect of increased dietary fiber should be evaluated before pharmaceutical intervention in the treatment of constipation in pregnancy (2).

Several oligosaccharides are reported to stimulate the growth of Bifidobacterium and improve the intestinal condition (3-6). Bifidobacterium provides strong support in major normal functions of the intestinal tract. The predominant growth of Bifidobacterium prevents constipation, antibiotic-associated diarrhea, and geriatric diseases, including cancer, in consequence of the suppression of intestinal putrefaction (7).

*To whom correspondence should be addressed.

E-mail: Yoshinobu_Kiso@suntory.co.jp
Xylooligosaccharides (XOS) are one of the most active oligosaccharides used for intestinal disorders. XOS is mainly composed of two or three xylose units with $\beta$ 1,4 linkages (Fig. 1). They are obtained by hemicellulose hydrolysis, which is relatively abundant in the cell walls of grains (8). XOS administration selectively increases intestinal Bifidobacterium and causes hardly any increase in other bacteria (9). Diarrhea decreased with the increase of intestinal Bifidobacterium by the daily intake of $1 \mathrm{~g}$ or $2.6 \mathrm{~g}$ of XOS (10), and the fecal water content was maintained within the normal range by $3.5 \mathrm{~g}$ XOS daily (9). Short chain fatty acids (especially acetic acid) in the feces of the subjects were increased and putrefactive products, such as $p$-cresol, indole and skatole, were decreased by the continual intake of $0.7 \mathrm{~g} / \mathrm{d}$ XOS (11). In a clinical study intended for adult women, the relative percentage of Bifidobacterium to total intestinal flora was significantly increased by the daily intake of $0.4 \mathrm{~g}$ of XOS, and stool frequency and abdominal conditions improved simultaneously (12).

In this study, we examined the efficay of XOS in preventing constipation in pregnant women.

\section{EXPERIMENT}

Subjects. The subjects were 30 constipated pregnant women at more than 23 wk' gestation who had not defecated for more than 5 consecutive days without laxatives. Patients suffering from complications were excluded. 


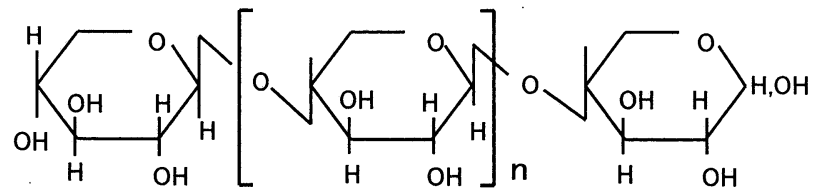

Fig. 1. Chemical structure of xylooligosaccharides.

Methods. The subjects were given $8 \mathrm{~g}$ of syrup containing $4.2 \mathrm{~g}$ XOS (Suntory Ltd., Osaka, Japan) daily for $4 \mathrm{wk}$. The syrup contained $75 \%$ of a solid component consisting of $70 \%$ oligosaccharides (biose, triose, etc.) and 30\% monosaccharides (mainly xylose), and was packed in identical tubes according to intake dosage. During the experimental period, the subjects maintained their regular diet, and their daily food intake was not restricted, although they refrained from taking laxatives and other oligosaccharide preparations.

The method of Kinugasa and Matsushita was used to evaluate the defecation data (13). During the study, clinical efficacy was assessed using a daily diary in which the subjects indicated the number of stools and the severity of the following symptoms: subjective amount of feces, stool consistency, stool color, stool smell, flatus, uncomfortable abdominal feeling, and abdominal pain. The subjects reported each item using a score. To assess stool consistency and color, the subjects were shown appearance standards. Their state of health such as appetite, amount of exercise, and skin condition were also assessed throughout the study. Approval of the Human Subjects Committee of the Kishiwada City Hospital was obtained before starting the study. Informed consent was obtained from all subjects to participate in the study.

Data analysis. Clinical efficacy was analyzed weekly for each subject during the experimental period. Statistical analysis was based on the SAS system. The number of stools was expressed as the means \pm SD, and compared statistically by one-way repeated-measures ANOVA. The Wilcoxon's signed rank test and $\chi$-square test were used to assess the defecation state. Values of $p$ less than 0.05 were considered significant.

\section{RESULTS}

\section{Pre-administration state of subjects}

Most of the subjects had taken laxatives and suffered uncomfortable symptoms of constipation. Twentyseven subjects defecated only once during the 2 -wk pretreatment period. Two subjects defecated 3 times and one subject defecated 5 times in the same period.

\section{Effect of XOS intake}

In all, 29 subjects completed the XOS administration schedule. One subject could not stop taking laxatives and failed to complete the study. She took a laxative on day 3 and gave up taking XOS syrup on the 7th day, and was excluded from the analysis.

During the XOS administration period, the symptoms of constipation decreased in all 29 subjects. The mean frequency of stools in the pre-treatment week was $1.1 \pm 0.4$ per week, increasing to $5.3 \pm 2.1$ in the first
A

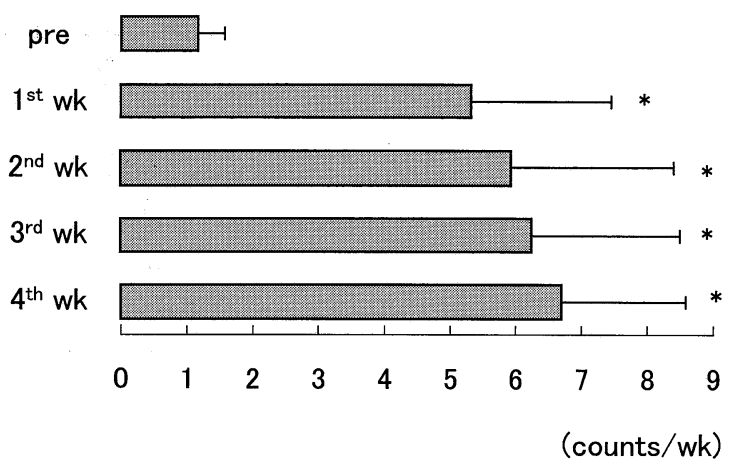

B

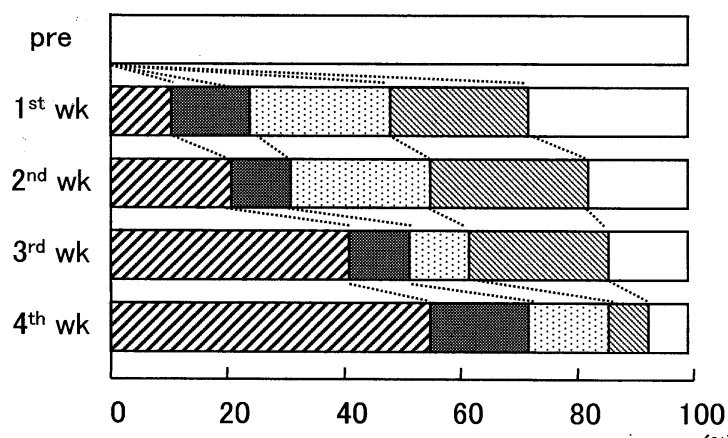

Fig. 2. Effects of xylooligosaccharide intake on the stool frequency of the subjects. Thirty pregnant women who had not defecated for more than $5 \mathrm{~d}$ without laxatives were given $4.2 \mathrm{~g}$ XOS daily for $4 \mathrm{wk}$. A: Weekly transition of the mean number of stools of the subjects. Data are presented as the means $\pm S D$. *Significant difference from the pre-treatment week, at $p<0.05$. B: Weekly transition of the subject ratio divided into groups by the number of days with defecation. Every day (ש্Z), $6 \mathrm{~d} / \mathrm{wk}$ (国), $5 \mathrm{~d} /$ wk (雨), $4 \mathrm{~d} /$ wk $(\mathbb{\mathbb { N }}), 3 \mathrm{~d} /$ wk or less $(\square)$.

week, $5.9 \pm 2.5$ in the second week, $6.2 \pm 2.2$ in the $3 \mathrm{rd}$ week, and $6.7 \pm 1.9$ in the 4th week as shown in Fig. $2 \mathrm{~A}$. Twenty-seven subjects were able to defecate spontaneously in the 4 th week (Fig. 2B).

When assessing the subjective amount of stools, 21\% of subjects reported "more than pre-treatment period" in the last week (Fig. 3A), but no significant change was noted from the first week. Almost all subjects reported that their feces became soft when taking XOS syrup. The frequency of very hard or hard stools decreased from the first week (36\%) to the 3rd week $(16 \%)$ and the 4th week (21\%) (Fig. 3B). No severe diarrhea was reported. The stool color changed from dark to yellowish brown. The indication of "yellowish" significantly increased from $7 \%$ in the first week to $21 \%$ in the last week (Fig. 3C). Each uncomfortable symptom decreased with improved defecation.

Of the 29 subjects who completed the experimental protocol, 15 (52\%) had improved defecation within $3 \mathrm{~d}$, 8 subjects (28\%) improved during days $4-7$, and 2 subjects $(7 \%)$ improved during the second week. At the end 
A

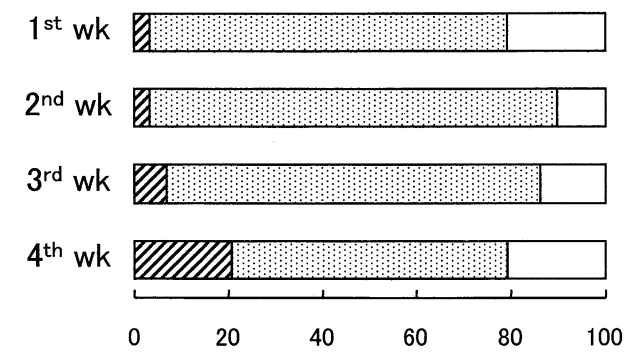

(\%)

B

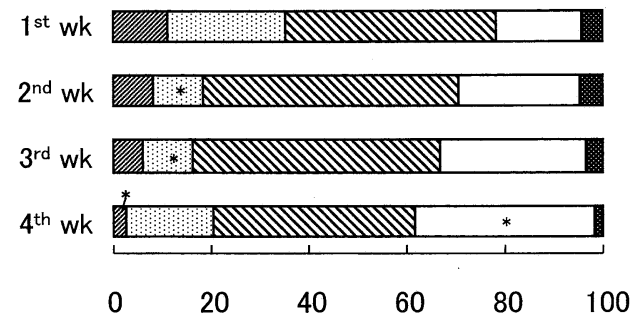

(\%)

C

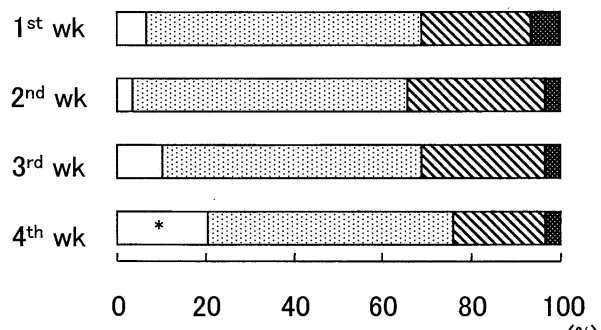

(\%)

Fig. 3. Effects of xylooligosaccharide intake on the patients' self-defined defecation symptoms. The subjects assessed their defecation symptoms throughout the experimental period using a score, and reported each item in a daily diary. To assess stool consistency and color, they were shown standards. A: Weekly transition of the subjective amount of feces: more than nor-

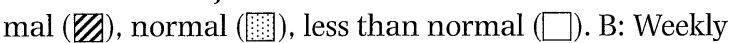

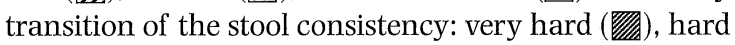

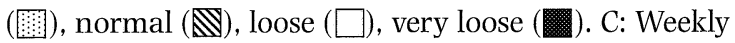
transition of the stool color: yellowish $(\square)$, yellowish brown (网), dark brown $(\mathbb{N})$, dark (1 $)$. *Significant difference from the first week, at $p<0.05$.

of the study, clinical symptoms such as inflation, a heavy abdominal feeling, discomfort, and abdominal pain disappeared in $70 \%$ of the subjects.

Throughout the experimental period, no side effects were reported or detected. The physical condition was good in all subjects during the experimental period, except for one who caught a cold and took medicine. After the experiment, childbirth and lactation were normal in all cases.

\section{DISCUSSION}

In this study, the intake of syrup containing $4.2 \mathrm{~g}$ of XOS improved constipation in pregnant women. The mean number of stools increased significantly, and most subjects were able to defecate spontaneously at the end of the study.

The occurrence of very loose or very hard stools decreased and the stool consistency normalized during the XOS intake period, suggesting that fecal water content tended to revert to within the normal range by XOS intake. These indications coincided with previous clinical studies, which established that the continual administration of XOS $(0.4-3.5 \mathrm{~g} / \mathrm{d})$ decreased the occurrence of diarrhea and stimulated bowel movements (9-11, 14).

The XOS intake dose in this study $(4.2 \mathrm{~g} / \mathrm{d})$ was more than in the previous experiments and corresponded to approximately half of the maximum non-affect dose of XOS (10). This was because the subjects had used laxatives habitually, and prompt defecation was necessary. The subjects were instructed to maintain their habitual revised diet throughout the experimental period, because it was thought inappropriate to impose dietary restrictions during the examination. They were in the latter stages of pregnancy and had already revised their food content based on nutritional guidance.

In the assessment of stool color, many subjects recognized a yellowish change of the stool color in the 3rd or 4 th week in this study. When the growth of Bifidobacterium is activated in the intestinal tract, the amount of fecal short chain fatty acids is commonly increased, reducing the stool $\mathrm{pH}$ and the yellowish stool color change, which reflects the environmental conditions in the intestine. It is thought that the growth of intestinal Bifidobacterium was activated by XOS administration as in previous studies, although the amount of flora was not directly examined in this study.

Stool frequency and stool consistency tended to change from the early stage of the experiment, before the stool color had become yellowish, indicating that constipation symptoms had improved partly through a physical function. Since XOS is a constituent of hemicellulose, which is a component of grain fibers, it might act as dietary fiber. It is thought that XOS improves the symptoms of constipation through two different functions: as a Bifidobacterium growth factor, and as a bulkforming agent.

Emollients, lubricants, hyperosmotics and stimulants are often prescribed to treat constipation. These agents facilitate defecation by acting directly on the intestine or compacted feces. Stimulant laxatives rarely cause abdominal cramping as they stimulate the colon. Loose stools in neonates are sometimes associated with the use of stimulant laxatives by breast-feeding women (2). The long-term use of laxatives occasionally leads to dependence, so they should be used carefully. XOS reaches the lower intestinal tract without being decomposed or absorbed in the upper intestinal tract (15), and is assimilated predominantly by Bifidobacterium (9). 
Bifidobacterium provides strong support in major normal functions of the intestinal tract (16), producing short chain fatty acids such as lactic acid and acetic acid. These acids lower the $\mathrm{pH}$ of the intestinal contents, inhibit the growth of putrefactive microorganisms, and assist in the physical removal of invading pathogens by stimulating intestinal peristalsis. In addition, there are other controversial claims for Bifidobacterium, such as its potential anti-tumor activity, its use as a treatment for antibiotic-associated diarrhea, its stimulation of the immune response, and the suppression of serum cholesterol levels.

Apart from these effects of XOS or Bifidobacterium on the intestinal contents, additional value is expected. For example, lipid metabolism and mineral absorption could be improved by the increased short chain fatty acids produced by Bifidobacterium. Imaizumi et al. reported that the elevation of serum cholesterol and triglyceride level was suppressed in rats fed XOS (17).

In conclusion, subjects with initial severe constipation were given $4.2 \mathrm{~g}$ of XOS daily for $4 \mathrm{wk}$. In most cases, the frequency of stools increased and the associated symptoms were reduced during the XOS intake period. It is supposed that XOS normalizes bowel movements by regulating intestinal flora and increasing the intestinal contents. XOS is a safe and effective intestinal conditioner suitable for pregnant women.

\section{REFERENCES}

1) Anderson AS. 1984. Constipation during pregnancy: Incidence and method used in its treatment in a group of Cambridgeshire women. Health Visitor 12: 363-364.

2) West T, Warren J, Cutts T. 1992. Diagnosis and management of irritable bowel syndrome, constipation, and diarrhea in pregnancy. Gastroenterol Clin North Am $\mathbf{2 1}$ : 793-801.

3) Gibson GR, Beatty ER, Wang X, Cumming JH. 1995. Selective stimulation of bifidobacteria in the human colon by oligofructose and inulin. Gastroenterology $\mathbf{1 0 8}$ : 975-982.

4) Ito M, Deguchi Y, Miyamori A. 1988. Effects of administration of galactooligosaccharides on the human fecal microflora, stool weight and abdominal sensation. Microb Ecol Health Dis 3: 285-292.
5) Hidaka H, Eida T, Takizawa T, Tokunaga T, Tashiro Y. 1986. Effects of fructooligosaccharides on intestinal flora and human health. Bifidobacteria Microflora 5: 3750.

6) Tanaka R, Takayama H, Morotomi M, Kuroshima T, Ueyama S, Matsumoto K, Kuroda A, Mutai M. 1983. Effects of administration of TOS and Bifidobacterium breve 4006 on the human fecal flora. Bifidobacteria Microflora 2: 17-24.

7) Mitsuoka T. 1990. Bifidobacteria and their role in human health. J Indust Microb 6: 263-268.

8) Koga K, Fujikawa S. 1993. Japanese Technology Reviews: Oligosaccharides; Production, Properties and Applications (Nakakuki T, ed), Vol 3, No 2, p 130-143. Gordon and Breach Science Publishers, Yverdon.

9) Okazaki M, Fujikawa S, Matsumoto N. 1990. Effect of xylooligosaccharide on the growth of Bifidobacteria. Bifidobacteria Microflora 9: 77-86.

10) Kobayashi T, Okazaki M, Fujikawa S, Koga K. 1991. Effect of xylooligosaccharides on feces of men. J Jpn Soc Biosci Biotech Agrochem 65: 1651-1653 (in Japanese).

11) Okazaki M, Fujikawa S, Matsumoto N. 1990. Effects of xylooligosaccharide on growth of Bifidobacteria. J Jpn Soc Nutr Food Sci 43: 395-401 (in Japanese).

12) Iino T, Nishijima $Y$, Sawada S, Sasaki H, Harada H, Suwa Y, Kiso Y. 1997. Improvement of constipation by a small amount of xylooligosaccharides ingestion in adult women. J Jpn Assoc Dietary Fiber Res 1: 19-24 (in Japanese).

13) Kinugasa A, Matsushita S. 1993. Effect of bifidobacterial material on abnormal evacuative symptoms. J New Remed Clin 42: 2181-2187 (in Japanese).

14) Fujikawa S, Okazaki M, Matsumoto N. 1991. Effect of xylo-oligosaccharide on growth of intestinal bacteria and putrefaction products. J Jpn Soc Nutr Food Sci 44: 37-40 (in Japanese).

15) Okazaki M, Koda H, Izumi R, Fujikawa S, Matsumoto N. 1991. In vitro digestibility and in vivo utilization of xylobiose. J Jpn Soc Nutr Food Sci 44: 41-44 (in Japanese).

16) Robinson RK, Samona A. 1992. Health aspects of 'bifidus' products: a revew. Int J Food Sci Nutr 43: 175-180.

17) Imaizumi K, Nakatsu $Y$, Sato M, Sedarnawati $Y$, Sugano M. 1990. Effect of xylooligosaccharide on blood glucose, serum and liver lipid and cecum short-chain fatty acids in diabetic rats. Agric Biol Chem 55: 199-205. 